\title{
Simulation of Wake Field Effects on High Energy Particle Beams *
}

\author{
R. Barlow, G. Kourevlev and A. Mercer, Manchester University, Manchester, UK and the Cockcroft Institute
}

\section{Abstract}

We present formulae for higher mode geometric intrabunch wakefields, such as will be produced in the ILC. These have been incorporated in the MERLIN code, and thus validated against existing data, and simple studies are done indicating the effect of wake fields in a typical ILC design.

\section{INTRODUCTION}

A bunch of charged particles induces currents in the beam pipe, and these lead to electromagnetic fields (wake fields) which affect the particles in the beam. This may have important consequences for the ILC, as particle bunches will be passing very close to collimators. This could lead to further deflection of off-axis bunches, or emittance dilution, and thus lowering of the luminosity.

Deviations from perfect conductivity and constant aperture lead to resistive and geometric wakefields. We consider only geometric wakefields, as the spoiler elements are relatively short. We consider only the effects of wake fields within a bunch, as the time between bunches is believed to be long enough for the wake currents to damp down.

For a bunch passing through an element the detailed motion within the element is not relevant, and we are interested in the deflection in angle of the particles that emerge from the element. If the bunch and the particle are on-axis then symmetry dictates that there is no effect: the effect can be expanded in powers of $r$. We use cylindrical coordinates $r, \theta, z$ and consider the effect on a particle, a distance $r$ from the centre, of a slice of thickness $d z$ of the bunch, preceding this particle by a distance $z$. Then $[1,2,3]$

$$
\begin{aligned}
& \bar{F}_{\perp}= \\
&-e \quad \sum_{m=1}^{\infty} W_{m}(z) m r^{m-1}\left[\hat { r } \left(Q_{m} \cos m \theta+\tilde{Q}_{m} \sin m \theta\right.\right. \\
&\left.-\hat{\theta}\left(Q_{m} \sin m \theta+\tilde{Q}_{m} \cos m \theta\right)\right]
\end{aligned}
$$

where

$$
\begin{gathered}
Q_{m}=\int_{0}^{2 \pi} d \theta^{\prime} \int_{0}^{\infty} d r^{\prime}\left(r^{\prime}\right)^{m+1} \operatorname{cosm} \theta^{\prime} \rho\left(r^{\prime}, \theta^{\prime}, z\right) \\
\tilde{Q}_{m}=\int_{0}^{2 \pi} d \theta^{\prime} \int_{0}^{\infty} d r^{\prime} r^{\prime m+1} \sin m \theta^{\prime} \rho\left(r^{\prime}, \theta^{\prime}, z\right)
\end{gathered}
$$

where $\rho(r, \theta, z)$ is the density of the bunch We assume, as in [4], that only $\theta^{\prime}=0$ need be considered, i.e. we consider deflection/distortion in a particular direction. This then simplifies to

* This work is supported in part by the Commission of the European Communities under the 6th Framework Programme "Structuring the European Research Area”, contract number RIDS-011899

$$
\begin{gathered}
F_{\perp, m}=-e W_{m} m r^{m-1} Q_{m} \\
Q_{m}=\int_{0}^{2 \pi} d \theta^{\prime} \int_{0}^{\infty} d r^{\prime} r^{\prime m+1} \rho\left(r^{\prime}, \theta^{\prime}\right)=Q(z) \overline{r^{m}}
\end{gathered}
$$

Where the moments $\overline{r^{m}}$ are known from the bunch distribution. $Q(z)$ is the charge of the slice, and the wake function for a steeply tapered collimator, moving from aperture $b$ to aperture $a$, is given by ([5])

$$
W_{m}(z)=2\left(\frac{1}{a^{2 m}}-\frac{1}{b^{2 m}}\right) e^{-m z / a} \Theta(z)
$$

where $\Theta(z)$ is a unit step function. The factor of 2 arises because one has to consider both sides of the collimator [6].

In the SLAC tests [7] the deflection of a particle bunch near a wall was measured. The effect of the wakefield is encompassed in the 'kick factor' $K$

$$
\Delta y^{\prime}=\frac{N r_{e}}{\gamma} K y
$$

\section{IMPLEMENTATION}

MERLIN already contains a 'wakefieldprocess' in collimator elements, however it only considers the first, linear ( $m=1$ ) mode. We extend this to $m=5$ and apply a deflection to a particle at radius $r_{i}$ in bin $i$

$\Delta y_{i}^{\prime}=-\frac{2 N r_{e}}{\gamma} \sum_{j=i+1}^{j=n b i n s} \sum_{m=1}^{5} \frac{m r_{i}^{m-1} Q\left(z_{j}\right) \overline{r_{j}^{m}}}{a^{2 m}} e^{-m\left(z_{j}-z_{i}\right) / a}$

where we have assumed $a<<b$. The code has been modfied by the addition of 4 extra terms to the Wakefieldprocess.

\section{VALIDATION}

We simulated the SLAC tests using a beam of energy $1.19 \mathrm{GeV}$, with $210^{10}$ elctrons, a bunch with $\epsilon_{x}=$ $0.36 \mathrm{~mm}, \epsilon_{y}=0.16 \mathrm{~mm}$, and $\sigma_{z}=0.65 \mathrm{~mm}$. We considered a square aperture of gap half-width $1.9 \mathrm{~mm}$. We took the lattice functions as $\beta_{x}=3 \mathrm{~m}, \beta_{y}=10 \mathrm{~m}$.

Figure 1 shows the predicted deflection when only the first $(m=1)$ term is included. It agrees with the data (Figure 2) for small displacements but, being linear, does not reproduce the non-linearity near the edge. However inclusion of 5 terms, as suggested by [4] on the basis of comparisons with the ECHO code, reproduces the data well (Figure3) even for beams close to the collimator wall. 


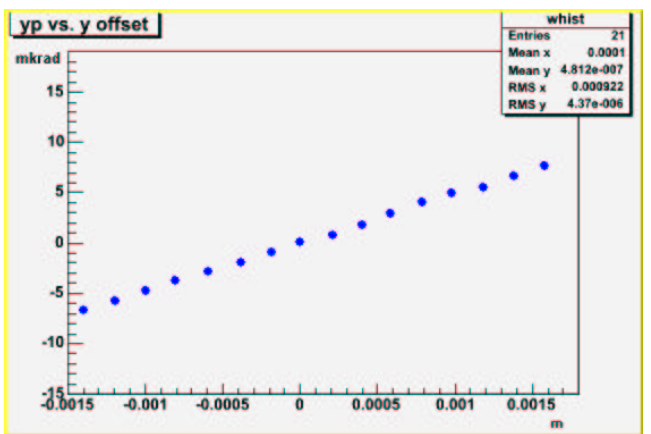

Figure 1: Predicted deflection using one term

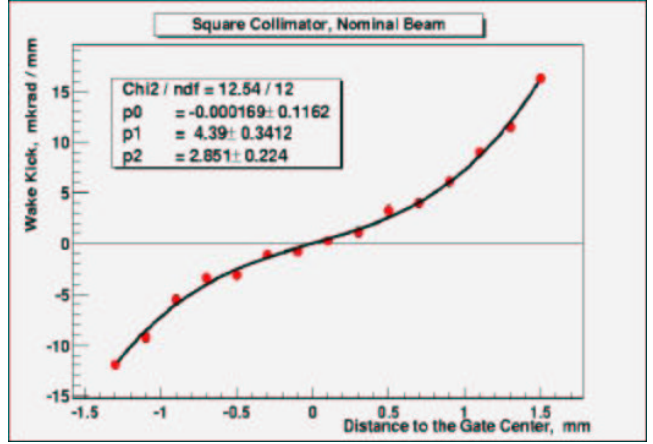

Figure 2: Data - from Ref[7]

\section{WAKE FIELDS IN THE TESLA BDS}

We use the TESLA BDS to investigate wake fields at a future linear collider, as optics files are available. These are read into MERLIN by the MADinterface code, which constructs the accelerator model. Wake potentials can be included at this stage. The TESLA collimation system consists of four betatron collimation systems spaced $45^{\circ}$ apart, preceded by an energy collimation system. The collimation system is a spoiler/absorber combination, however wakefield effects from the absorbers are generally much smaller than those from the spoilers [8] so we consider only the spoiler wakefields. In this simulation any particle striking the spoiler is simply removed.

We consider wake field effects on the core bunch, and also on the halo. For the core bunch we take standard

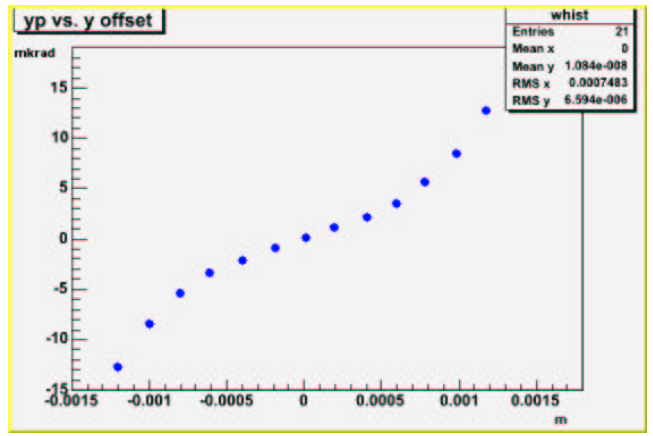

Figure 3: Predicted deflection using 5 terms
Gaussian distributions. At present we only consider the first $m=1$ term, which is good for small deviations from the axis.

We considered a standard bunch which was $0.01 \mathrm{~mm}$ off axis in $y$ (which is large: around $3 \sigma$ ) at the exit from the accelerator. Figure 4 shows the distribution in $y$ at the IP with no wakefields simulated, and Figure 5 shows the distribution with wake fields included. The difference is discernible but not large: the rms spread is increased, the tail to negative $y$ is larger. The reduced number is caused by a few particles hitting a collimator. Overall the effect appears small, and not a serious problem for the feasibility of the accelerator.

We used version 8.02 (June 26,2000) of the optics. Preliminary examination of the more recent 8.05 gave effects which were even smaller.

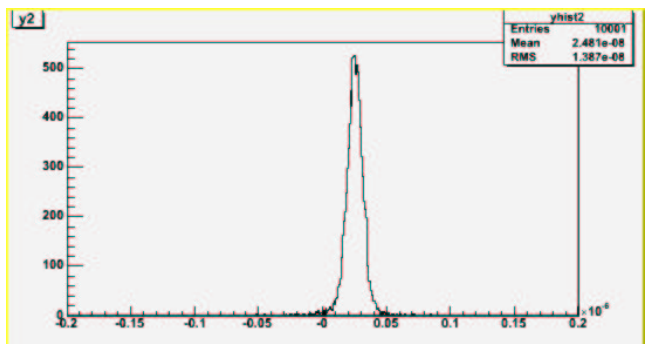

Figure 4: $y$ position at IP without wake fields

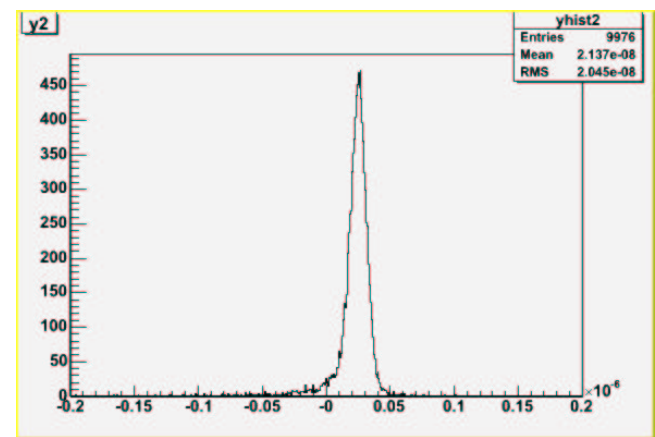

Figure 5: $y$ position at IP with wake fields

These are early results, and need to be substantiated by more detailed studies and cross-checks.

\section{CONCLUSIONS}

We have a formalism for including wake fields in Merlin which reproduces the existing data and can be used for Linear Collider studies: the first such studies indicate that the effects are small.

\section{REFERENCES}

[1] K. Yokoya, 'Impedance of slowly tapered structures', CERN report SL/90-88 (AP) (1990) 
[2] G. Stupakov, 'Geometric Wake of a smooth taper', SLACPUB-95-7086 (1995)

[3] G. Stupakov, 'Geometric Wake of a smooth flat collimator', SLAC-PUB-95-7167 (1996)

[4] I. Zagorodnov, T.1 Weiland, M. Dohlus and M Körfer 'Nearwall Wakefields for Optimize Geometry if te TTF-2 COllimator', TESLA report 2003-23

[5] P. Raimondi, F. J. Decker, T. Useher and C. K. Ng. 'Closed form expression for the Geometric effect of a Beam Scraper on the Transverse Beam Distribution', PAC 97 (Vancouver) and SLAC-PUB-8552

[6] F. Zimmermann, K. L. F. Bane, C. K. Ng, 'Collimator Wake Fields in the SLC Final Focus', EPAC 96 (BArcelona), SLAC-PUB-7137

[7] D. Onoprienko, 'Measurements of Transverse Wakefields of Tapered Collimators' in LC02, SLAC, February 2003

[8] P. Tenenbaum, 'Collimator Wakefield Calculation for ILCTRC Report', SLAC LCC-0101, (2002)

[9] A. Drozhdin et al, 'Comparison of the TESLA, NLC and CLIC beam collimation system performace', FERMILABTM-2200, (2003) 